\title{
Depth-integrated flow modelling taking into account bottom curvature
}

\section{Modélisation d'un écoulement intégré en profondeur avec prise en compte de la courbure du fond}

\author{
B.J. DEWALS, Research Fellow of the National Fund for Scientific Research, Laboratory of Applied Hydrodynamics \\ and Hydraulic Constructions (HACH), University of Liege, Chemin des Chevreuils, 1 Bat B52/3+1, Belgium. \\ Tel.: +32 (0)4 36695 96; fax: +32(0)4 36695 58; e-mail: B.Dewals@ulg.ac.be
}

\begin{abstract}
S. ERPICUM, Professor's Assistant, Laboratory of Applied Hydrodynamics and Hydraulic Constructions (HACH), University of Liege, Chemin des Chevreuils, 1 Bat B52/3+1, Belgium. Tel.: +32 (0)4 36695 96; fax: +32 (0)4 3669558 ; e-mail: S.Erpicum@ulg.ac.be
\end{abstract}

\begin{abstract}
P. ARCHAMBEAU, Research Engineer, Laboratory of Applied Hydrodynamics and Hydraulic Constructions (HACH), University of Liege, Chemin des Chevreuils, 1 Bat B52/3+1, Belgium. Tel.: +32 (0)4 36695 96; fax: +32 (0)4 3669558 ; e-mail: Pierre.Archambeau@ulg.ac.be
\end{abstract}
S. DETREMBLEUR, Research Engineer, Laboratory of Applied Hydrodynamics and Hydraulic Constructions (HACH), University of Liege, Chemin des Chevreuils, 1 Bat B52/3+1, Belgium. Tel.: +32 (0)4 36695 96; fax: +32 (0)4 3669558 ; e-mail: Sylvain.Detrembleur@ulg.ac.be
M. PIROTTON, Professor, Laboratory of Applied Hydrodynamics and Hydraulic Constructions (HACH), University of Liege, Chemin des Chevreuils, 1 Bat B52/3+1, Belgium. Tel.: +32 (0)4 36695 96; fax: +32 (0)4 36695 58; e-mail: Michel.Pirotton@ulg.ac.be

\begin{abstract}
Successfully modelling flows over a spillway and on strongly vertically curved bottoms is a challenge for any depth-integrated model. This type of computation requires the use of axes properly inclined along the mean flow direction in the vertical plane and a modelling of curvature effects. The proposed generalized model performs such computations by means of suitable curvilinear coordinates in the vertical plane, leading to a fully integrated approach. This means that the flows in the upstream reservoir, on the spillway, in the stilling basin and in the downstream river reach are all handled in a single simulation. The velocity profile is generalized in comparison with the uniform one usually assumed in the classical shallow water equations. The pressure distribution is modified as a function of the bottom curvature and is thus not purely hydrostatic. Representative test cases, as well as the application of the extended model to the design of a large hydraulic structure in Belgium, lead to satisfactory validation results
\end{abstract}

\section{RÉSUMÉ}

Modéliser avec succès les écoulements au-dessus d'un déversoir et sur des fonds fortement incurvés verticalement est un défi pour n'importe quel modèle intégré en profondeur. Ce type de calcul exige l'utilisation d'axes correctement inclinés le long du sens d'écoulement moyen dans le plan vertical et de modéliser les effets de la courbure. Le modèle généralisé proposé exécute de tels calculs au moyen de coordonnées curvilignes appropriées dans le plan vertical, menant à une approche entièrement intégrée. Ceci signifie que les écoulements dans le réservoir amont, sur le déversoir, dans le bassin d'amortissement et dans tout le fleuve à l'aval sont traités dans une même simulation. Le profil de vitesse est généralisé par comparaison avec le profil uniforme habituellement considéré dans les équations classiques en eau peu profonde. La distribution de pression est modifiée en fonction de la courbure du fond et n'est donc pas purement hydrostatique. Les cas tests représentatifs, aussi bien que l'application du modèle général à la conception d'une grande structure hydraulique en Belgique, donnent des résultats de validation satisfaisants.

Keywords: Shallow water, finite volume, curvilinear coordinates, spillway, reservoir, numerical model.

\section{Introduction}

Dams have been built for thousands of years to control floods, to exploit water as hydropower, to provide fresh water for irrigation, for drinking and to enable other economic activities. At the beginning of the third millennium, large dams produce one fifth of the world's electricity (World Commission on Dams, 2000). Furthermore, they still play an essential role through their water supply function and are seen as strategic elements in ensuring long-term sustainable development. Achieving efficient and

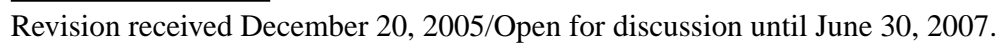


economical designs for dams and other hydraulic structures thus constitutes an issue of continually growing interest for a wide range of hydro-practitioners and decision makers.

Experimental modelling has been considered for decades as the single tool for the design of hydraulic structures. Whenever appropriate similitude laws are applied, scale models provide the modeller with reliable results. Though attractive for highly complex configurations (e.g. aerated or sediment-laden turbulent flows), such experimental tests suffer from high cost and weak flexibility.

Today numerical models are becoming steadily more available and supply results with an ever increasing level of confidence. They have become more attractive tools for predicting a wide scope of flow behaviours and are considered a credible alternative to classical scale models for a large set of applications.

While fully 3D models are still barely possible to exploit for large-scale simulations, modelling flows over vertically curved bottoms remains a challenging task for any depth-integrated model. Such a computation requires the use of axes properly inclined along the local mean flow direction in the vertical plane. Since this local mean flow direction changes over the computation domain, a suitable modelling of the vertical curvature effects is needed.

The herein presented 2D generalized model handles correctly those effects by means of suitable curvilinear coordinates in the vertical plane. This frame of reference is chosen in such a way that one of the two curvilinear axes follows the local bottom curvature. Hence the set of generalized shallow water equations includes explicit expressions of channel bottom slope, but also channel vertical curvature. It also takes into consideration the derivative of the channel curvature. The velocity profile is different from the uniform one usually assumed in the classical shallow water equations. The pressure distribution is modified to take into account the bottom curvature and is thus not purely hydrostatic.

Following a brief literature review, the paper presents a description of the new depth-averaged model, with detailed developments and applications. Described applications include validation test cases, as well as a large-scale study for the design of an important hydraulic structure in Belgium. Both cases lead to reasonable agreement between numerical results and validation data.

\section{Literature review}

The first attempt to introduce vertical curvature in a shallowwater model was performed by Dressler (1978). He developed a 1D model on a similar idea but without any integration on the water depth. His mathematical formulation was thus non-conservative. Later he presented a modified friction term (Dressler and Yevjevich, 1984), taking into account the uneven velocity profile.

Experimental validations of this first model were performed by Sivakumaran et al. (1981, 1983) and later by Ilhan (1994). A 2D generalization, as well as a finite element computer code to solve the model, were then proposed by Berger and Carey (1998a,b). Simultaneously, 3D models were applied to spillway overflows (e.g. Bürgisser and Rutschman, 1999).

Other authors (Khan and Steffler, 1996a,b) have modelled flows over vertically curved beds by means of vertically averaged and moment equations. However, this method presents the drawback of involving eight equations in 1D and ten equations in $2 \mathrm{D}$, in comparison with, respectively, two and three equations in the present case.

\section{Model description}

\subsection{General aim}

The primary purpose of the computation model described is to achieve a unified 2D modelling of the flows upstream, downstream and on river training structures (e.g. a spillway) in one single simulation.

The large majority of flows occurring in rivers can reasonably be seen as shallow and characterized by relatively small vertical velocity components everywhere, except near local singularities. Those singularities, such as weirs or spillways, can however play a major role in the flow behaviour around the structure, but also in the far field upstream and downstream.

This confirms the relevance of extending the shallow-water equations (SWE), which simply assume the bed curvature and its rate of change to be negligible. The SWE approach may thus be preferred only if no sharp change in the bed inclination occurs. Though these conditions are very widely met in waterways, numerical results demonstrate the necessity for a better approximation for spillway overflows.

In the SWE model, the only assumption states that velocities normal to a main flow direction are smaller than those in the main flow direction (Pirotton, 1994). As a consequence, the pressure field is found to be almost hydrostatic everywhere and an easy integration of the local conservation laws can be completed over the water depth. In the new extended model, a similar depthaveraging operation is performed in a frame of reference with curvilinear lines of coordinates following the main flow direction. As a consequence, the new set of equations includes terms involving not only the channel inclination $\theta$, but also containing explicitly the bottom curvature $\kappa$ and its rate of change $\kappa^{\prime}$.

\subsection{2 conceptual and mathematical model}

In this section, the 2D formulation of the model is first described and its mathematical properties then investigated. Bed curvature is considered in one direction $(x)$.

\subsubsection{Curvilinear frame of reference}

The fundamental idea of the extended model is to replace the Cartesian coordinates $(x, z)$ in the vertical plane with curvilinear coordinates $(\xi, \eta)$ selected in order that:

- the curvilinear abscissa $\xi$ follows the local channel bottom;

- the second curvilinear axis of reference $(\eta)$ is normal to the local bed direction; 


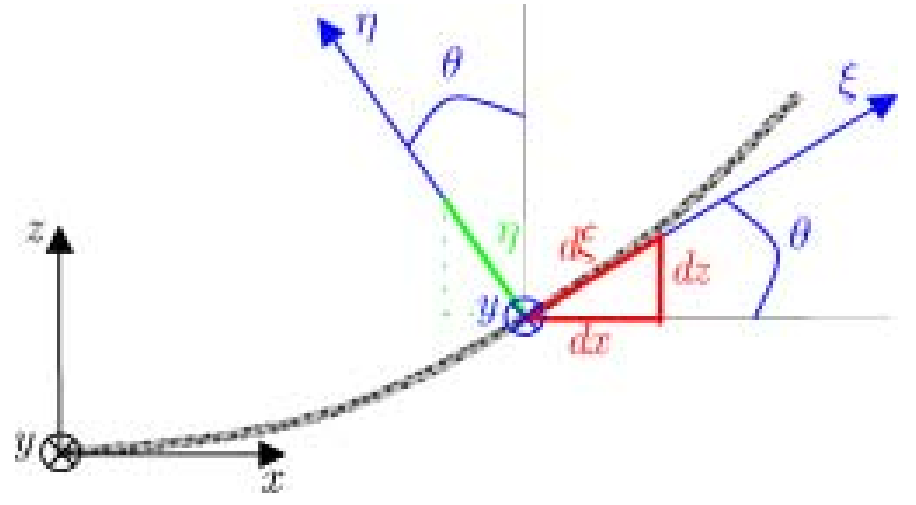

Figure 1 Symbols and axes of reference for the 2D model.

- the third axis of reference ( $y$ ) is simply normal to the plane of curvature.

Figure 1 illustrates the new axes of reference and the main notations used.

In order to allow the evaluation of the space derivatives involved in the curvature expression and those present in the conservation equations, the mathematical function describing the channel bed has to be continuous, have a continuous slope $(\theta)$ and a continuous curvature $(\kappa)$. The curve representing the bottom must therefore be three times differentiable.

\subsubsection{Change of variables}

The correspondence between the Cartesian coordinates and their curvilinear counterparts is given by the following relations:

$$
\begin{aligned}
& x=x(\xi, \eta)=x_{\text {bed }}(\xi)-\eta \sin \theta, \\
& y=y, \\
& z=z(\xi, \eta)=z_{\text {bed }}(\xi)+\eta \cos \theta .
\end{aligned}
$$

The unit vector tangent to the channel bottom in a vertical plane is defined as

$$
\vec{t}=\left(\begin{array}{c}
\frac{\mathrm{d} x_{\mathrm{b}}}{\mathrm{d} \xi} \\
\frac{\mathrm{d} y_{\mathrm{b}}}{\mathrm{d} \xi} \\
\frac{\mathrm{d} z_{\mathrm{b}}}{\mathrm{d} \xi}
\end{array}\right)=\left(\begin{array}{c}
\cos \theta \\
0 \\
\sin \theta
\end{array}\right)
$$

Hence the Jacobian matrix can be easily deduced from relations (1) to (3):

$$
\mathbf{J}=\left[\begin{array}{ccc}
\cos \theta(1-\kappa \eta) & 0 & -\sin \theta \\
0 & 1 & 0 \\
\sin \theta(1-\kappa \eta) & 0 & \cos \theta
\end{array}\right],
$$

where $\kappa$ stands for the bottom curvature defined as

$$
\kappa=\left(\vec{t} \wedge \frac{\mathrm{d} \vec{t}}{\mathrm{~d} \xi}\right) \cdot \vec{e}_{y}=\frac{\mathrm{d} \theta}{\mathrm{d} \xi} .
$$

The Jacobian of the variables change is calculated as the determinant of matrix $\mathbf{J}$ :

$$
\operatorname{det}(\mathbf{J})=J=1-\kappa \eta \text {. }
$$

The fundamental relation between derivatives in both systems of reference reads:

$$
\left(\begin{array}{c}
\frac{\partial}{\partial x} \\
\frac{\partial}{\partial y} \\
\frac{\partial}{\partial z}
\end{array}\right)=\left[\begin{array}{ccc}
\cos \theta(1-\kappa \eta)^{-1} & 0 & -\sin \theta \\
0 & 1 & 0 \\
\sin \theta(1-\kappa \eta)^{-1} & 0 & \cos \theta
\end{array}\right]\left(\begin{array}{c}
\frac{\partial}{\partial \xi} \\
\frac{\partial}{\partial y} \\
\frac{\partial}{\partial \eta}
\end{array}\right) .
$$

A change of variables is relevant provided that the corresponding Jacobian remains positive. In the case of a negative bed curvature (convex), no special restriction needs to be introduced. On the other hand, if the bed curvature is positive (concave), the coordinate $\eta$ must be limited to a value depending on the sharpness of the curvature. The Jacobian expression above remains positive as long as the water height verifies the following condition (with $\kappa>0$ and $R$ the radius of curvature):

$$
H<\frac{1}{\kappa}=R \text {. }
$$

\subsubsection{Velocity transformation and velocity profile}

The velocity vector $\vec{v}$ may be expressed by its components in each frame of reference:

$$
\vec{v}=U \vec{e}_{\xi}+V \vec{e}_{y}+W \vec{e}_{\eta}=u \vec{e}_{x}+v \vec{e}_{y}+w \vec{e}_{z} .
$$

Those components are related by the simple transformation formula

$$
\left(\begin{array}{c}
U \\
V \\
W
\end{array}\right)=\left[\begin{array}{ccc}
\cos \theta & 0 & \sin \theta \\
0 & 1 & 0 \\
-\sin \theta & 0 & \cos \theta
\end{array}\right]\left(\begin{array}{c}
u \\
v \\
w
\end{array}\right) .
$$

Following the work by Dressler (1978), the most straightforward assumption concerning velocity distribution has been selected. This corresponds to a non-rotational flow in the vertical plane:

$$
\operatorname{rot} \vec{v} \cdot \vec{e}_{y}=0
$$

Translating the above differential relation into curvilinear coordinates, one obtains:

$$
\frac{\partial W}{\partial \xi}=J \frac{\partial U}{\partial \eta}-\kappa U
$$

Finally, heeding the shallowness of the flow, the corresponding $\xi$-velocity profile is given by:

$$
U(\xi, y, \eta, t)=\frac{C(\xi, y, t)}{1-\kappa \eta},
$$

with $C$ representing the longitudinal velocity along the channel bottom. The profile of the velocity is hence completely determined by the condition of irrotationality (11) and the bottom curvature (Fig. 2).

\subsubsection{Transformation of a general PDE}

A general conservation law, such as

$$
\frac{\partial s}{\partial t}+\frac{\partial f_{x}}{\partial x}+\frac{\partial f_{y}}{\partial y}+\frac{\partial f_{z}}{\partial z}=S,
$$

may be transformed as a function of the curvilinear coordinates thanks to the fundamental relation (8). This operation leads to a 


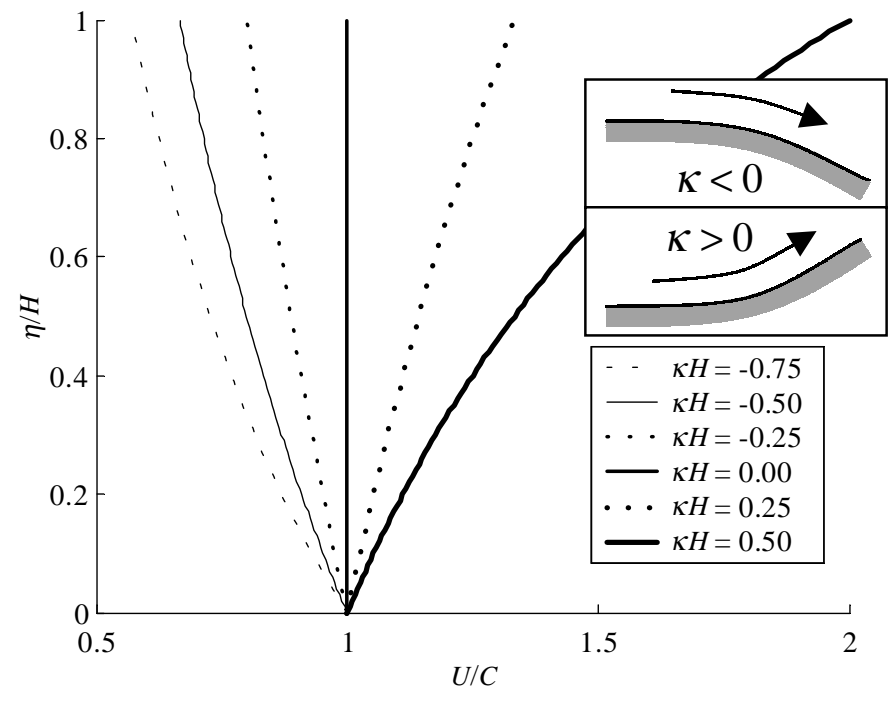

Figure 2 Velocity profiles for different values of the non-dimensional curvature.

new conservative law

$$
\begin{gathered}
\frac{\partial}{\partial t}(J S)+\frac{\partial}{\partial \xi}\left(f_{x} \cos \theta+f_{z} \sin \theta\right)+\frac{\partial}{\partial y}\left(J f_{y}\right) \\
+\frac{\partial}{\partial \eta}\left(f_{z} J \cos \theta-f_{x} J \sin \theta\right)=J S
\end{gathered}
$$

which can now be used to transform the flow mass and momentum balances.

\subsubsection{Local conservation laws for the flow}

The local continuity equation can be easily deduced from its Cartesian form and the general relation (15):

$$
\frac{\partial U}{\partial \xi}+\frac{\partial}{\partial y}(J V)+\frac{\partial}{\partial \eta}(J W)=0 .
$$

The same transformation performed with the momentum conservation equations leads to three new equations, which can be combined to provide the local momentum conservation laws expressed in curvilinear coordinates:

$$
\begin{gathered}
\frac{\partial}{\partial t}(J U)+\frac{\partial}{\partial \xi}\left(U^{2}+\frac{p}{\rho}\right)+\frac{\partial}{\partial y}(J U V) \\
+\frac{\partial}{\partial \eta}(J U W)=-g J \sin \theta+\kappa U W, \\
\frac{\partial}{\partial t}(J V)+\frac{\partial}{\partial \xi}(U V)+\frac{\partial}{\partial y}\left[J\left(V^{2}+\frac{p}{\rho}\right)\right] \\
+\frac{\partial}{\partial \eta}(J V W)=0
\end{gathered}
$$

and

$$
\begin{gathered}
\frac{\partial}{\partial t}(J W)+\frac{\partial}{\partial \xi}(U W)+\frac{\partial}{\partial y}(J V W)+\frac{\partial}{\partial \eta}\left[J\left(W^{2}+\frac{p}{\rho}\right)\right] \\
=-g J \cos \theta-\kappa\left(U^{2}+\frac{p}{\rho}\right) .
\end{gathered}
$$

According to the classical assumption regarding the shallowness of the flow $\left(W^{2} /\left(U^{2}+V^{2}\right) \ll 1\right)$, the purely advective terms expressing an acceleration perpendicular to the bottom may be ignored in the latter equation. A complete non-dimensional

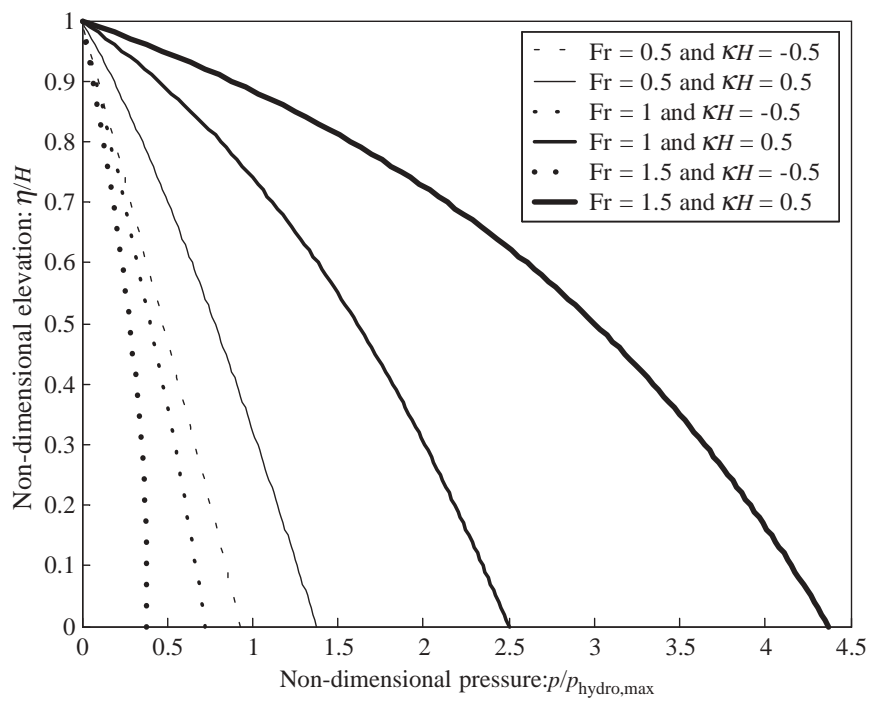

Figure 3 Generalized pressure distributions for various Froude numbers and non-dimensional curvatures $\kappa H$.

analysis of order of magnitude has been performed and demonstrates that this conclusion remains valid for the model expressed in curvilinear coordinates. As a result, the generalized pressure distribution can be deduced directly by integrating relation (19) along the $\eta$-axis:

$$
\frac{p(\eta)}{\rho}=g \cos \theta(H-\eta)+\frac{C^{2}}{2}\left[\frac{1}{(1-\kappa H)^{2}}-\frac{1}{(1-\kappa \eta)^{2}}\right] .
$$

It can be easily verified that in the case of a zero-curvature bed, the term in square brackets vanishes in relation (20) and hence the hydrostatic pressure distribution is restored. Figure 3 illustrates the effect of bed curvature on the modified pressure field as a function of Froude number, defined as

$$
\operatorname{Fr}=C / \sqrt{g H \cos \theta} .
$$

The figure highlights a higher sensitivity of the pressure profile to positive curvatures, rather than to negative ones.

\subsubsection{Boundary conditions}

Kinematic boundary conditions apply at the free surface and at the bottom.

The interface between the studied liquid and the ambient air is defined by the impossibility of the fluid crossing this surface. This implies an equality between the velocity of the fluid in the direction normal to the interface and the velocity of this interface in the same normal direction. According to Whitham (1974), and taking into consideration the specific velocity profile of the flow over a vertically curved bed, this equality of normal velocities can be expressed by:

$$
\begin{aligned}
W(t, \xi, y, H)= & \frac{\partial H}{\partial t}+\frac{U(t, \xi, y, H)}{1-\kappa H} \frac{\partial H}{\partial \xi} \\
& +V(t, \xi, y, H) \frac{\partial H}{\partial y} .
\end{aligned}
$$


The impermeability of the bottom is obviously expressed by:

$W(t, \xi, y, 0)=0$.

Since the free surface position is not known a priori, its location constitutes an additional unknown and calls for a second boundary condition. This dynamic boundary condition simply states that the pressure at the free surface is identical to the atmospheric pressure, selected as the reference:

$p(\xi, y, H, t)=p_{\text {atm }}=0$.

Choosing a non-zero value for the atmospheric pressure would not provoke any change in the final conservation equations.

If the bottom pressure becomes lower than the atmospheric pressure, two phenomena can potentially occur. Either the flow is aerated and the flow layer is likely to detach from the bottom, or there is no aeration and a risk of cavitation arises. Those features can occur when the relative pressure at the bottom reaches zero or negative values:

$$
\frac{p_{\mathrm{b}}}{\rho}=g H \cos \theta+C^{2} \kappa H \frac{1-\kappa H / 2}{(1-\kappa H)^{2}} \leq 0 .
$$

If the bottom curvature remains positive, the model never predicts either cavitation or detachment. On the other hand, with $\kappa<0$, expression (25) predicts negative relative pressures in the case where the following condition is fulfilled (see Fig. 4):

$$
\frac{\frac{p_{\text {atm }}}{\rho g H \cos \theta}+1}{|\kappa| H \frac{1+|\kappa| H / 2}{(1+|\kappa| H)^{2}}}<\frac{C^{2}}{g H \cos \theta} .
$$

\subsubsection{Depth-averaged equations for the flow}

Integrating Eq. (16) from the bottom $(\eta=0)$ to the free surface $(\eta=H$ ) leads to the depth-averaged mass conservation for a flow over an arbitrarily curved bed:

$$
\frac{\partial \mathcal{H}}{\partial t}+\frac{\partial q}{\partial \xi}+\frac{\partial}{\partial y}(\mathcal{H} V)=0
$$

with $\mathcal{H}$ representing the actual "volume" in a cell:

$$
\mathcal{H} \triangleq H \bar{J}=H\left(1-\frac{\kappa H}{2}\right)
$$

and $q$ the specific discharge defined as

$$
q(\xi, t)=H \bar{U}=\int_{0}^{H(\xi, t)} U(\xi, \eta, t) \mathrm{d} \eta .
$$

The previous expression can be evaluated given the particular velocity profile (13):

$$
q=H C \frac{\ln (1-\kappa H)}{-\kappa H} .
$$

A similar procedure applied to Eqs (17) and (18) leads to the momentum conservation along the $\xi$-axis:

$$
\begin{aligned}
& \frac{\partial}{\partial t}(H C)+\frac{\partial}{\partial \xi}\left(\frac{H C^{2}}{1-\kappa H}\right)+\frac{\partial}{\partial y}(H C V) \\
& +\frac{\partial}{\partial \xi}\left[g \frac{H^{2}}{2} \cos \theta+\frac{H C^{2}}{2} \frac{\kappa H}{(1-\kappa H)^{2}}\right] \\
& =-g H \sin \theta+\kappa H \overline{U W}
\end{aligned}
$$

and the corresponding relation along the $y$-axis:

$$
\frac{\partial}{\partial t}(\mathcal{H} V)+\frac{\partial}{\partial \xi}(V q)+\frac{\partial}{\partial y}\left(\mathcal{H} V^{2}+H \overline{J \frac{p}{\rho}}\right)=0,
$$

with the mean pressure term written out in full as follows:

$$
\begin{aligned}
H J \frac{p}{\rho}= & g \frac{H^{2}}{2} \cos \theta\left(1-\frac{\kappa H}{3}\right) \\
& +\frac{H C^{2}}{2} \frac{1-\frac{\kappa H}{2}}{(1-\kappa H)^{2}}-\frac{H C^{2}}{2} \frac{\ln (1-\kappa H)}{-\kappa H} .
\end{aligned}
$$

It can be easily verified that in the case of a zero curvature bed, Eqs (27), (31) and (32) become identical to the set of the classical 2D shallow water equations.

According to the non-dimensional analysis of orders of magnitude, the term $\kappa H \overline{U W}$ in (31) may not necessarily be ignored. It can be accounted for thanks to a proper assumption regarding the profile of velocities $W$ perpendicular to the bottom. This issue is addressed in Section 3.2.9 of the present paper.

Viewing Eqs (27), (31) and (32), it is clear that the system remains conservative, which is a crucial asset for numerical discretization.

A bottom friction term can be added on the right-hand side of Eqs (32) and (33). Dressler and Yevjevich (1984) suggest a proper formulation of classical empirical friction laws (Chezy, Manning) for a 1D model including bed curvature. In addition, in further developments of the present research, the velocity profile (13) will be enhanced to take into account the corresponding bottom shear stress.

\subsubsection{Mathematical properties}

This section shows that the extended shallow water equations remain hyperbolic for a wide range of parameters. Different flow regimes are also distinguished.

The 1D correspondence of the set of equations to be solved can be written as follows:

$$
M \frac{\partial \vec{s}}{\partial t}+\frac{\partial \vec{f}}{\partial x}=\vec{S}
$$

with

$$
\begin{aligned}
& M=\left(\begin{array}{cc}
1-\kappa H & 0 \\
0 & 1
\end{array}\right) \text { and } \vec{s}=\left(\begin{array}{c}
s_{1} \\
s_{2}
\end{array}\right)=\left(\begin{array}{c}
H \\
H C
\end{array}\right) ; \\
& \vec{f}=\left(\begin{array}{c}
f_{1} \\
f_{2}
\end{array}\right)=\left(\begin{array}{c}
q(H, H C) \\
\frac{H C^{2}}{1-\kappa H}+g \cos \theta \frac{H^{2}}{2}+\frac{H C^{2} \kappa H}{2(1-\kappa H)^{2}}
\end{array}\right) .
\end{aligned}
$$

The source term is given by

$$
\vec{S}^{\mathrm{T}}=(0 \quad-g \mathcal{H} \sin \theta+\kappa H \overline{U W}) .
$$

Hence the characteristic matrix $A$ of the system is evaluated by means of the following operation:

$$
A=M^{-1}\left(\begin{array}{ll}
\frac{\partial f_{1}}{\partial s_{1}} & \frac{\partial f_{1}}{\partial s_{2}} \\
\frac{\partial f_{2}}{\partial s_{1}} & \frac{\partial f_{2}}{\partial s_{2}}
\end{array}\right),
$$


which finally leads to

$$
A=\left(\begin{array}{cc}
C \frac{\kappa H+(1-\kappa H) \ln (1-\kappa H)}{\kappa H(1-\kappa H)^{2}} & \frac{-\ln (1-\kappa H)}{\kappa H(1-\kappa H)} \\
g H \cos \theta-C^{2} \frac{1-\kappa H(3-\kappa H)}{(1-\kappa H)^{3}} & C \frac{2-\kappa H}{(1-\kappa H)^{2}}
\end{array}\right) .
$$

It can be easily shown that the limit of expression (39) for weak curvatures is equivalent to the characteristic matrix of the classical shallow water equations.

Determining the eigenvalues $\lambda$ of matrix $A$ can be achieved by finding the roots of the polynomial:

$$
\tilde{\lambda}^{2}-2 \operatorname{Fr} b \tilde{\lambda}+\left(\mathrm{Fr}^{2}-1\right) c,
$$

where non-dimensional eigenvalues are defined as

$$
\tilde{\lambda}=\frac{\lambda}{\sqrt{g H \cos \theta}} .
$$

The new coefficients $b$ and $c$ in the polynomial (40) are, respectively, given by

$$
b=\frac{\kappa H(3-\kappa H)+(1-\kappa H) \ln (1-\kappa H)}{2 \kappa H(1-\kappa H)^{2}}
$$

and

$$
c=\frac{\mathrm{Fr}^{2} \kappa H(2-\kappa H)+\mathrm{Fr}^{2} \ln (1-\kappa H)+(1-\kappa H)^{3} \ln (1-\kappa H)}{\kappa H\left(\mathrm{Fr}^{2}-1\right)(1-\kappa H)^{4}} .
$$

Both of those expressions equal simply unity in the case of a vanishing curvature.

The hyperbolic nature of the extended SWE model is preserved provided that two real roots can be found for the polynomial (40). As shown in Fig. 4, those two real solutions exist for moderate Froude numbers on a convex bottom and whatever the Froude number on a concave bottom.

A zero bottom pressure occurs before the set of equations becomes elliptic (see Fig. 4).

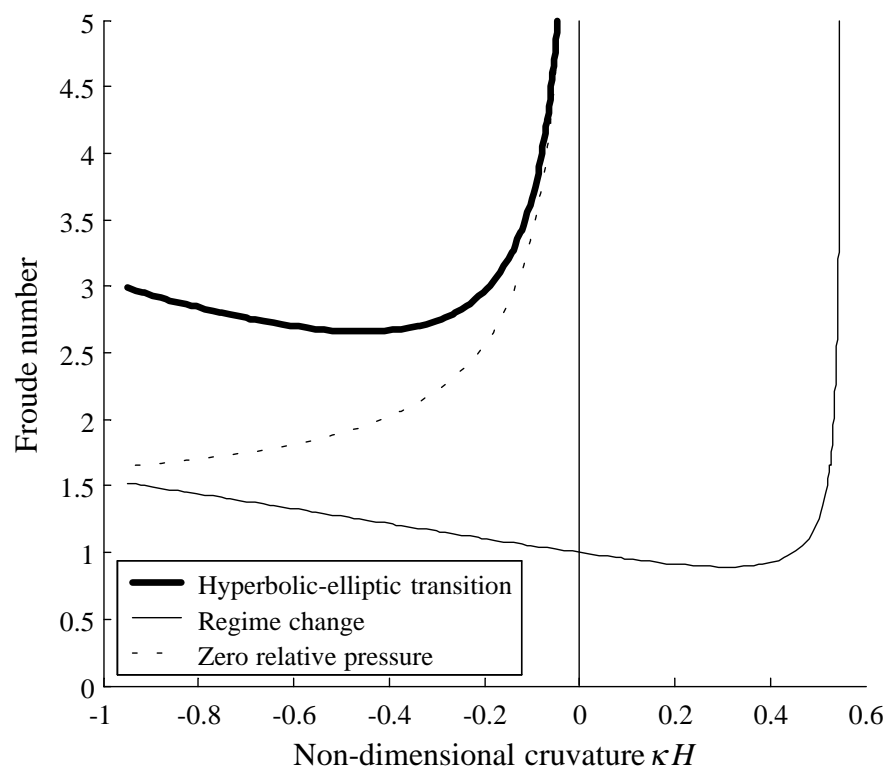

Figure 4 Curves of hyperbolic-elliptic transition, flow regime change and zero relative pressure.
Two flow regimes can be identified. The subcritical regime corresponds to one characteristic in each direction. The supercritical regime corresponds to two characteristics oriented towards downstream. The curve of flow regime change decreases down to a minimum close to 0.9 and then increases very fast, so much so that it is impossible to change regime for $\kappa H$ higher than 0.54 .

\subsubsection{Velocity component along $\eta$-axis}

The aim of this section is to evaluate the term $\kappa H \overline{U W}$, neglected until now in the momentum balance. The local mass conservation equation (16) can be integrated from the bottom to any elevation $\eta \leq H$, leading to an explicit expression for the velocity component $W$ :

$$
\begin{aligned}
W(\eta)= & \frac{1}{1-\kappa \eta} \frac{\partial}{\partial \xi}\left[C \frac{\ln (1-\kappa \eta)}{\kappa}\right] \\
& -\frac{1}{1-\kappa \eta} \eta\left(1-\frac{\kappa \eta}{2}\right) \frac{\partial V}{\partial y} .
\end{aligned}
$$

This expression fulfils both kinematic boundary conditions. Indeed, according to (44), the velocity $W$ remains zero at the bottom and equals the value given by (22) at the free surface. Simplified to the 1D case, the result (44) is also in accordance with those published by previous authors (Dressler, 1978; Berger and Carey, 1998a).

For a vanishing bed curvature, the velocity profile (44) corresponds to an almost linear distribution, like in a Boussinesq model:

$$
W(\eta)=-\eta\left[\frac{\partial C}{\partial \xi}+\frac{\partial V}{\partial y}\right]+O(\kappa H)
$$

and it deviates significantly from this distribution for stronger bed curvatures.

As a result, the corresponding term in the momentum balance becomes:

$$
\begin{aligned}
\int_{0}^{H}(\kappa U W) \mathrm{d} \eta= & \frac{C}{\kappa} \frac{\partial C}{\partial \xi} \frac{\kappa H+\ln (1-\kappa H)}{(1-\kappa H)} \\
& -\frac{\kappa^{\prime}}{\kappa^{2}} C^{2}\left[\frac{\kappa H(2-\kappa H)}{2(1-\kappa H)^{2}}+\frac{\ln (1-\kappa H)}{1-\kappa H}\right] \\
& -H C \frac{\partial V}{\partial y} \frac{\kappa H}{2(1-\kappa H)} .
\end{aligned}
$$

\subsection{Algorithmic implementation}

\subsubsection{Space discretization}

A finite volume scheme is used for space discretization in order to ensure an optimum mass and momentum conservativity, which are prerequisites for handling properly discontinuous solutions, such as hydraulic jumps.

\subsubsection{Flux evaluation and boundary conditions}

An original Flux Vector Splitting (FVS) is used for the space discretization of the complete set of equations (Mouzelard, 2002). This second order upwind scheme is Froude independent. Its stability has been demonstrated through a theoretical study of the SWE mathematical system, as well as a von Neumann stability analysis. 
Several expressions in the additional terms of the extended model present a very stiff behaviour for low-value curvatures. For this reason, expressions, such as the specific discharge definition (30) or the mean pressure term (33), are replaced in the numerical model by a series expansion as soon as $\kappa H$ becomes very small. In expression (46) again, a series expansion will be preferred if the curvature is low. Hence a proper numerical behaviour is preserved and no accuracy losses arise.

The models allow the user to specify any inflow discharge as an upstream boundary condition and possibly a water height in the case of a supercritical inflow. The downstream boundary condition can be a free surface elevation, a water height, a Froude number or even no specified condition, if the outflow regime is supercritical.

\subsubsection{Other features}

The herein described model is a part of the WOLF software package, developed in the Division of Applied Hydrodynamics and Hydraulic Construction $(\mathrm{HACH})$ at the University of Liege. WOLF includes a set of complementary modules for simulating free surface flows: process-oriented hydrology (Archambeau et al., 2001), 1D and 2D hydrodynamic models (Archambeau et al., 2002) possibly involving sediment transport (Dewals et al., 2002) or air entrainment (André et al., 2003), as well as inverse modelling tools (Bollaert et al., 2002).

A user-friendly interface makes the pre- and post-processing operations very convenient and straightforward to control. The grid generator deals with multiblock 2D structured meshes. The interface enhances post-processing capabilities, including $2 \mathrm{D}$ and $3 \mathrm{D}$ views, as well as animations.

The multiblock organization of the computation code allows the activation of the extended equations only in the vicinity of the spillway. So, the simple SWE are solved in the rest of the whole computation domain. This possibility leads to CPU time savings.

\section{Validation and case study}

This section aims at providing a precise assessment of the extent of the improvements enabled by the generalized SWE model in terms of results accuracy and realism.

The section includes a well illustrated comparison between the classical SWE and the extended model for simple test cases. Full details of the experimental conditions can be found in the original reference cited for each example.

CPU time requirements and achieved accuracy are compared for both numerical models.

Furthermore, we describe a case study of a stilling basin design based on the new model.

\subsection{Standard spillway}

\subsubsection{Description}

Recommendations from the US Bureau of Reclamation (1987) for a standard spillway have been exploited to define the geometry and flow conditions of the simulation. The studied standard spillway is described by

$$
\frac{z}{H_{0}}=-K\left(\frac{x}{H_{0}}\right)^{n},
$$

with $H_{0}$ representing the standard head. The local bed slope and curvature can be immediately deduced from the previous relation. The complete spillway geometry is inferred from an abacus supplied by the US Bureau of Reclamation (1987). In the present case, $K=0.507$ and $n=1.854$ were used. Subsequently the local slope $\theta$ and curvature $\kappa$ have been deduced.

Given a specific discharge $q=1.36 \mathrm{~m}^{2} / \mathrm{s}$, the corresponding head has been evaluated thanks to the Rehbock formula (Sinniger, 1989): $H_{0}=0.81 \mathrm{~m}$.

\subsubsection{Results}

Empirical formulae, corroborated by 2D-vertical simulations performed with WOLF (Mouzelard, 2002), give the following value for the upstream water elevation measured above the crest: $h_{0}=0.78 \mathrm{~m}$.

Figure 5 shows that the upstream water elevation is slightly overestimated by the depth-averaged approach. Nevertheless, using the extended model including the curvature effects reduces the error by a factor of three (see Table 1).

Note that the extended model has been used on the spillway section, while the SWE model is solved in the upstream zone.

\subsection{Symmetric profile}

Sivakumaran et al. (1983) studied the flow over a symmetric profile in a flume of $9.15 \times 0.75 \times 0.445 \mathrm{~m}$. The flume roughness corresponds to a Manning coefficient $n \approx 0.01 \mathrm{~m}^{-1 / 3} \mathrm{~s}$ and experiments were carried out with two different specific discharges: 0.11197 and $0.03599 \mathrm{~m}^{2} / \mathrm{s}$.

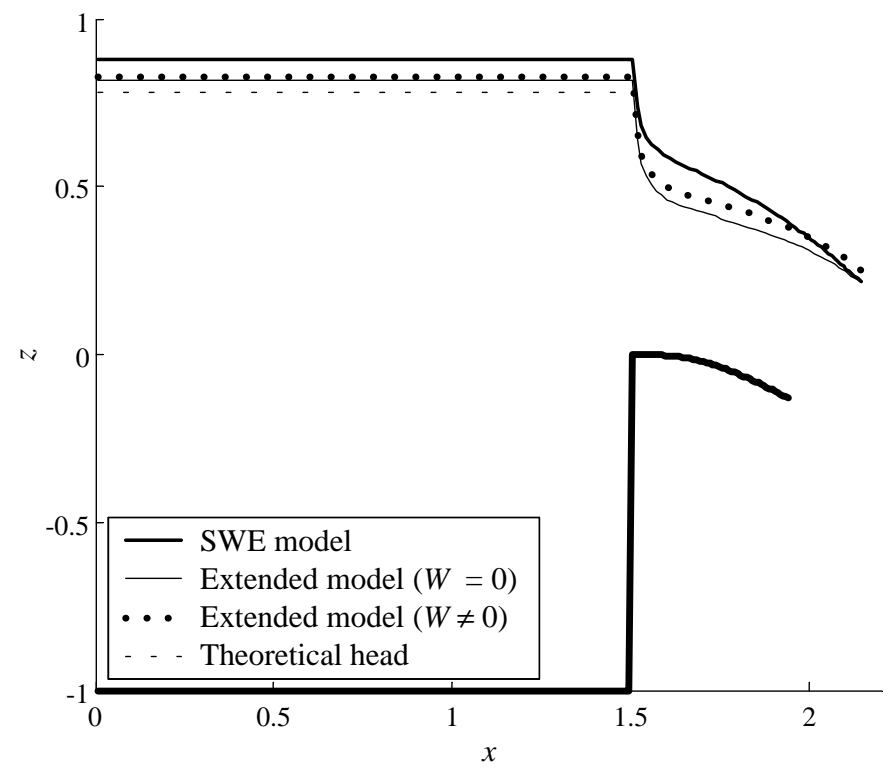

Figure 5 Standard spillway computed with the classical SWE model and the extended one. The influence of the velocity component along $\eta$ is illustrated. 
Table 1 Model results for the standard spillway

\begin{tabular}{llc}
\hline Model & Upstream level $(\mathrm{m})$ & Error \\
\hline 2DV & 0.78 & - \\
Classical SWE & 0.878 & $13 \%$ \\
Curvilinear $(W=0)$ & 0.815 & $4 \%$ \\
Curvilinear $(W \neq 0)$ & 0.819 & $5 \%$ \\
\hline
\end{tabular}
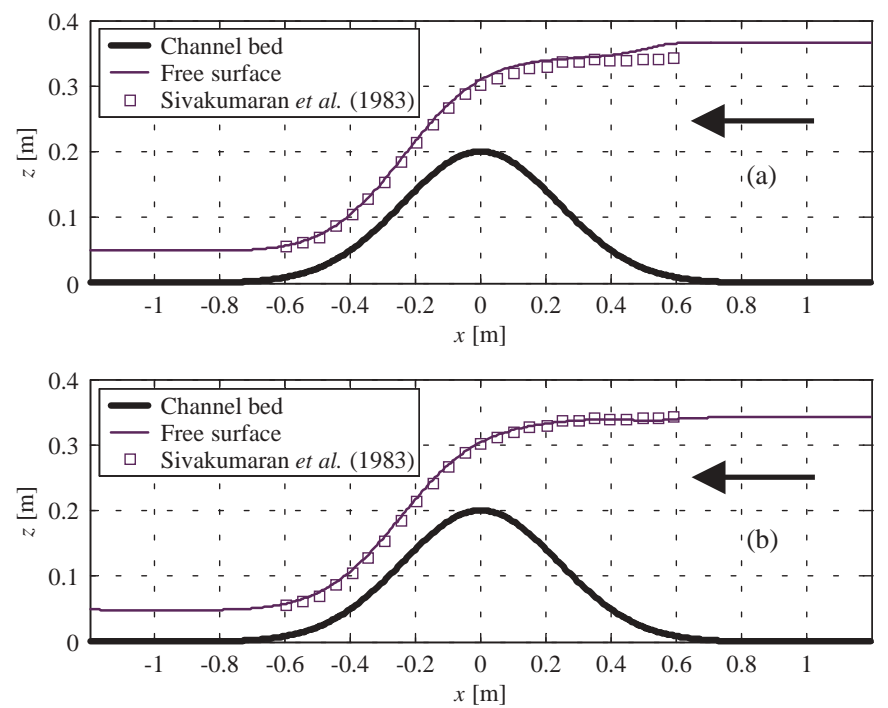

Figure 6 Water surface for the symmetric profile of Sivakumaran et al. (1983), simulated with the SWE (a) and with the extended model (b).

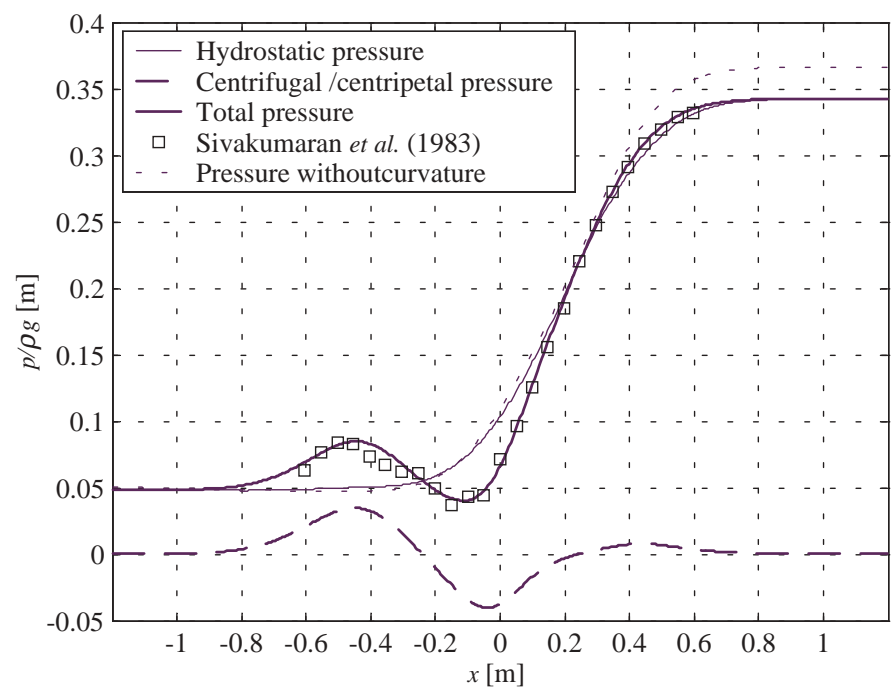

Figure 7 Bed pressure for the symmetric profile of Sivakumaran et al. (1983).

In both cases, the present extended model with curvature leads to significantly more accurate predictions for the free surface location, as well as for the bed pressure. Results for the higher discharge are presented in Figs 6 and 7.

\subsection{Flip-bucket spillway}

Water profile and bed pressure measurements, conducted on a laboratory flip-bucket spillway, were presented by Khan and Steffler (1996a). The slope of the chute was $20^{\circ}$ and the idealized flipbucket was represented by a circular arc of radius $15.4 \mathrm{~cm}$. Two specific discharges were tested: 0.0187 and $0.0292 \mathrm{~m}^{2} / \mathrm{s}$.

The same Manning coefficient as in the previous simulations was applied. Figure 8 shows a comparison between observed and computed pressures on the bottom of the spillway for the higher discharge. The pressure profile computed with the herein presented model agrees well with the measurement.

\subsection{Design of the stilling basin of the Nisramont dam in Belgium}

\subsubsection{Introduction}

Located on the upstream part of the Ourthe River in Belgium, the 21-m high Nisramont dam was built in 1958 and was to be used at first as a cofferdam during the construction of a large dam downstream. But this second large dam was never built and Nisramont became a definitive reservoir of $3 \mathrm{hm}^{3}$, providing the drinking water supply to a large part of the population of the south of Belgium and producing hydroelectricity, while supporting lowwater level discharges of the Ourthe River (Fig. 9).

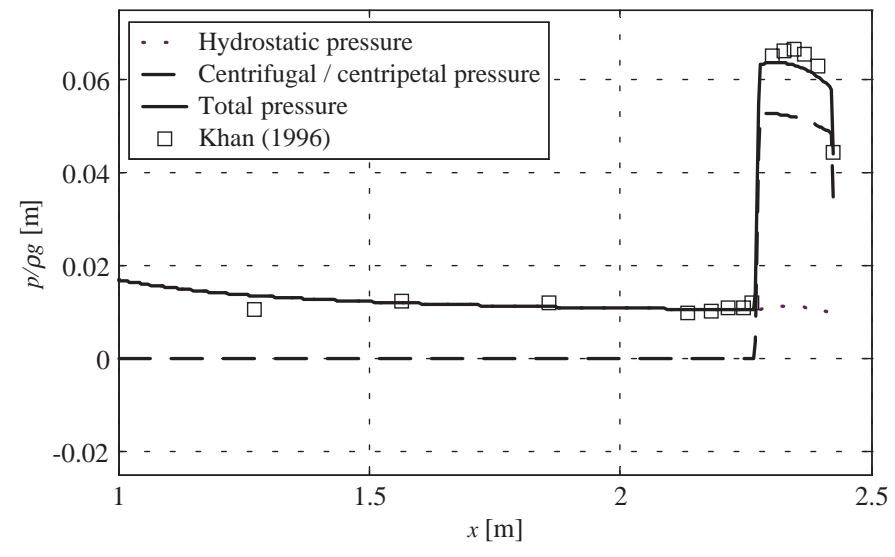

Figure 8 Bed pressure on the flip-bucket spillway tested by Khan and Steffler (1996a).

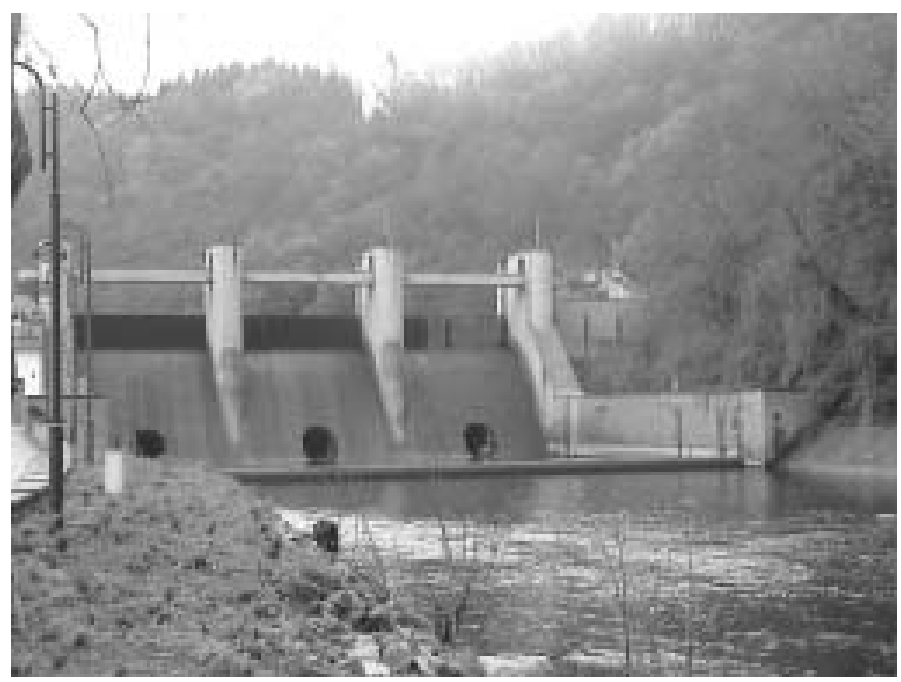

Figure 9 Downstream view of the Nisramont dam (@ $\mathrm{HACH})$. 
Due to its temporary primary tasks, the stilling basin downstream of the three 12.5-m large bays of the crest spillway was under-designed.

In order to be sure of the structure and following climate change observations, the Ministère wallon de l'Equipement et des Transport (MET) - Direction des barrages de l'Est (D. 241), entrusted the HACH with evaluating up to date critical flood discharges and designing a new stilling basin able to deal with probable floods.

After validation of the existing situation and for extreme observed events, the new model presented was used to verify the new design of the stilling basin.

\subsubsection{Simulation features}

The domain of simulation covered $700 \mathrm{~m}$ of the Ourthe River, $50 \mathrm{~m}$ upstream and $650 \mathrm{~m}$ downstream of the dam crest. Thanks to the new numerical model, the whole of the structure was modelled in a single process.

Upstream discharge boundary conditions were deduced from a large-scale simulation of the whole of the reservoir. Downstream, data of a limnimeter was used as a water level boundary condition.

General topographic information was available on a $1-\mathrm{m}$ square grid and came from aerial laser measurements (MET). The main river bed topography was interpolated by WOLF from cross section profiles available every metre. All this information was integrated in WOLF and automatically interpolated on a regular $25 \mathrm{~cm}$ square grid. Then the geometry and the topography of the dam were manually integrated from paper plans.

In order to minimize CPU time while keeping good accuracy in the critical part of the domain, four different blocks were defined for the computation. The mesh size was $25 \mathrm{~cm}$ in the upstream reservoir and on the dam and stilling basin, while it was $1 \mathrm{~m}$ on the downstream part, as shown in Fig. 10. The new model was exclusively used in block 2, while the classical SWE model of WOLF2D was applied in the other blocks.

All the simulations and results used were realized with WOLF package.

\subsubsection{Results}

Simulation of the well-documented $175 \mathrm{~m}^{3} / \mathrm{s}$ flood of 3 January 2003 leads to a very good agreement between

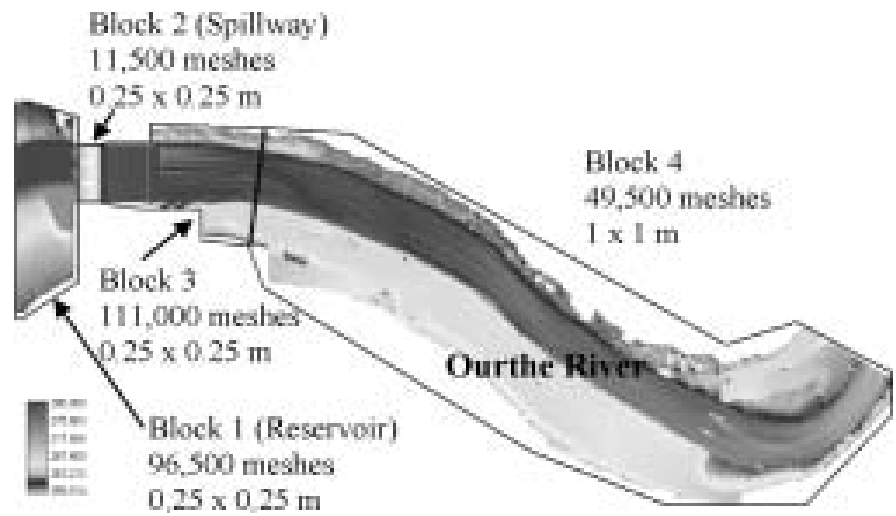

Figure 10 Shaded aerial view of the topography used for the simulation. numerical results and observations of the real event (Fig. 11).

For example, the position of the hydraulic jump at the downstream extremity of the stilling basin is reliable in order to make a comparison (Fig. 12).

On the basis of photos, the real position of the flow discontinuity was more or less $31 \mathrm{~m}$ downstream of the spillway toe. The numerical result was $29 \mathrm{~m}$ (Fig. 13).

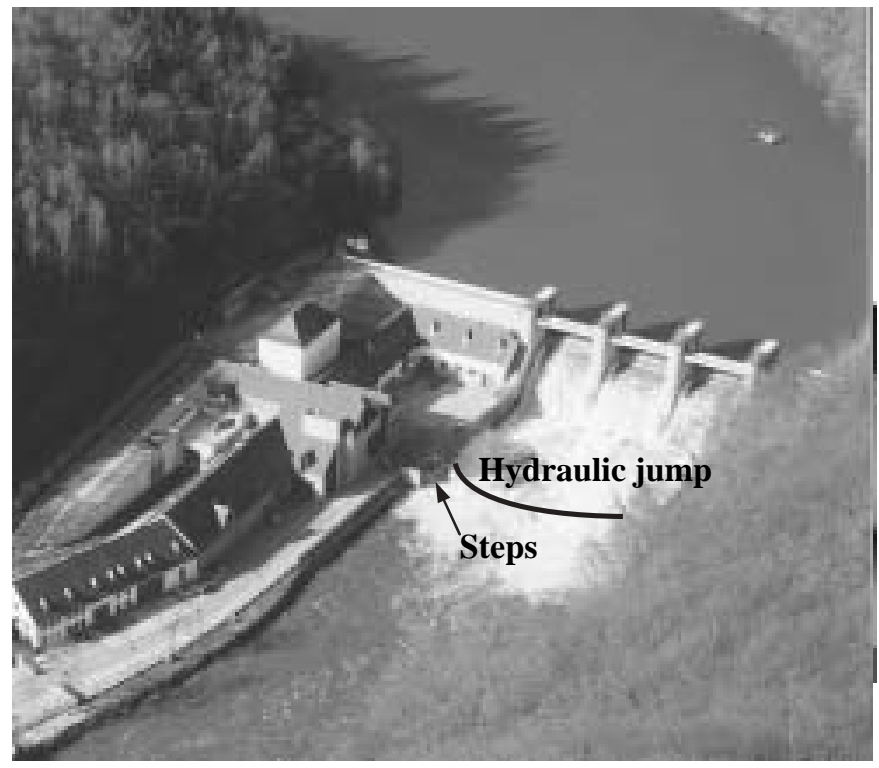

Figure $11 \quad 175 \mathrm{~m}^{3} / \mathrm{s}$ flood on the spillway (@ MET).

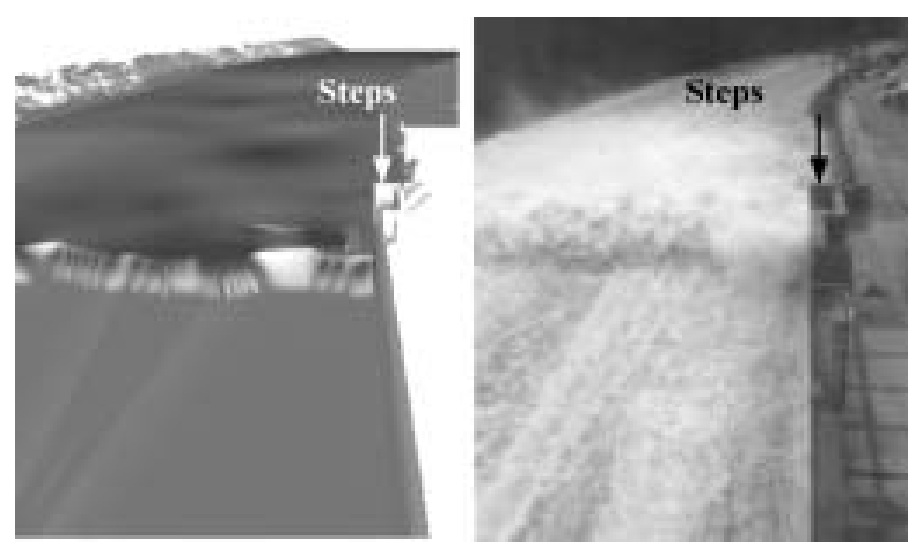

Figure 12 Comparison of the hydraulic jump location in the stilling basin for a $175 \mathrm{~m}^{3} / \mathrm{s}$ flood.

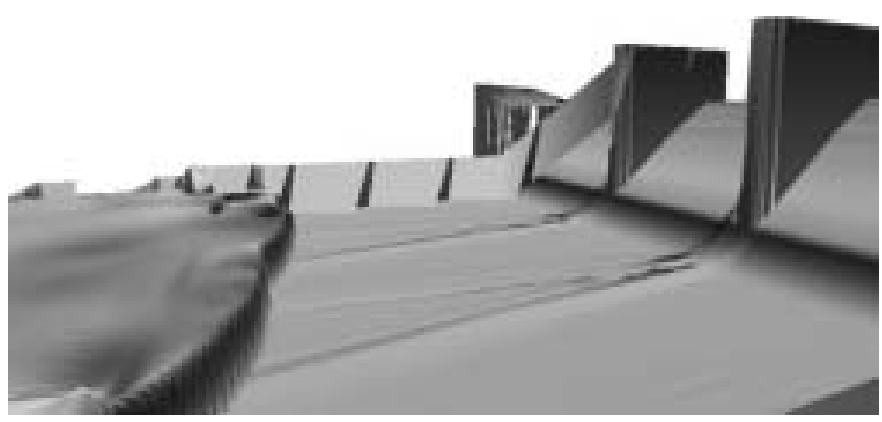

Figure $133 \mathrm{D}$ view of the numerical computation of a $175 \mathrm{~m}^{3} / \mathrm{s}$ flood on the spillway. 
Cross waves shape and length were compared, as well as dried areas at the spillway piles bottom and the reservoir water elevation.

Moreover, water levels reached in the river downstream of the dam were in good agreement with those measured in reality.

Following this first validation and calibration stage, at the end of which Manning's coefficient was found to be equal to 0.014 and 0.037 , respectively, for concrete areas and natural river, the numerical model was used to verify the modified design of the stilling basin, for the design flood of $340 \mathrm{~m}^{3} / \mathrm{s}$.

This led to a good agreement between predicted and computed reservoir water levels, and to confirmation of the effective behaviour of the new structure.

\section{Conclusion}

The model presented generalizes the shallow water theory by incorporating effects of streamline vertical curvature. It leads to better estimations of particle velocity profiles and nonhydrostatic pressure distribution over curved surfaces. More accurate upstream water elevation can thus be predicted.

The approach enables simulations completely integrating the flow behaviour in the vicinity of large river structures such as dams. The flows in the upstream reservoir, on the spillway, in the stilling basin and in the downstream river reach, can be treated in one single process-oriented simulation.

After a literature review, the full development of the model has been described and its mathematical properties have been analysed. Several successful test cases have been described and a field application to a stilling basin design has also been presented.

\section{Acknowledgements}

We acknowledge the assistance of the Ministère wallon de l'Equipement et des Transport (MET)_Direction des barrages de l'Est (D. 241), which owns the Nisramont dam study results and authorized their publication.

\section{Notation}

$C=$ value at the channel bottom of the velocity component along $\xi$-axis $\left(\mathrm{LT}^{-1}\right)$

$\vec{e}_{i}=$ unit vector along $i$-axis $(-)$

$f_{i}=$ general flux vector along $i$-axis

$\mathcal{H}=$ first conservative unknown (L)

$H=$ water height $(\mathrm{L})$

$h_{0}=$ theoretical water level, measured above the crest, upstream of the standard spillway (L)

$H_{0}=$ head upstream of the standard spillway (L)

$J=$ Jacobian of the variables transformation (-)

$K=$ coefficient of the standard spillway formula (-)

$n=$ exponent of the standard spillway formula (-)

$p=$ pressure $\left(\mathrm{ML}^{-1} \mathrm{~T}^{-2}\right)$

$p_{\text {atm }}=$ atmospheric pressure $\left(\mathrm{ML}^{-1} \mathrm{~T}^{-2}\right)$ $q=$ specific discharge along $\xi$-axis $\left(\mathrm{L}^{2} \mathrm{~T}^{-1}\right)$

$R=$ radius of curvature $(\mathrm{L})$

$\vec{s}=$ vector of arbitrary unknowns

$s_{i}=$ arbitrary unknown

$S=$ general source term

$t=$ time $(\mathrm{T})$

$u=$ velocity component along $x$-axis $\left(\mathrm{LT}^{-1}\right)$

$U=$ velocity component along $\xi$-axis $\left(\mathrm{LT}^{-1}\right)$

$v=$ velocity component along $y$-axis $\left(\mathrm{LT}^{-1}\right)$

$\vec{v}=$ fluid velocity vector $\left(\mathrm{LT}^{-1}\right)$

$V=$ velocity component along $y$-axis $\left(\mathrm{LT}^{-1}\right)$

$w=$ velocity component along $z$-axis $\left(\mathrm{LT}^{-1}\right)$

$W=$ velocity component along $\eta$-axis $\left(\mathrm{LT}^{-1}\right)$

$x=$ Cartesian abscissa in the plane of curvature $(\mathrm{L})$

$y=$ coordinate normal to the plane of curvature $(\mathrm{L})$

$z=$ Cartesian elevation in the plane of curvature $(\mathrm{L})$

$\eta=$ curvilinear coordinate normal to the bed (L)

$\theta=$ bed slope angle $(-)$

$\kappa=$ bottom curvature $\left(\mathrm{L}^{-1}\right)$

$\kappa^{\prime}=$ rate of change of the bed curvature $\left(\mathrm{L}^{-2}\right)$

$\rho=$ fluid specific mass $\left(\mathrm{ML}^{-3}\right)$

$\xi=$ curvilinear coordinate along the bed (L)

Over bar $=$ depth-averaged value

\section{References}

1. André, S., Dewals, B.J., Pirotton M. and Schleiss, A. (2003). "Quasi 2D-numerical Model of Aerated Flow Over Stepped Chutes". In: PRINOS, P. (ed.), Proc. 30th IAHR Congress. IAHR: Thessaloniki, Greece, pp. 671-678.

2. Archambeau, P., ERpicum, S, Mouzelard T. and PIRotTon, M. (2001). "Impact Studies and Water Management with WOLFHYDRO: A New Physically Based Hydrological Solver". In: Proc. Int. Symposium on Environmental Hydraulics. Arizona State University, USA.

3. Archambeau, P., Dewals, B, Detrembleur S, Erpicum, S. and PirotTon, M. (2003). "A Set of Efficient Numerical Tools for Floodplain Modeling”. In: UIJTTEWAAL, W.S.J. (ed.), Shallow Flows, IfH \& TU Delft, Delft, pp. 141-158.

4. Berger, R.C. and CAREY, G.F. (1998a). "Free-surface Flow Over Curved Surfaces-Part I: Perturbation Analysis”. Int. J. Numer. Meth. Fluids 28(2), 191-200.

5. BERGER, R.C. and CAREY, G.F. (1998b). "Free-Surface Flow Over Curved Surfaces-Part II: Computational Model”. Int. J. Numer. Meth. Fluids 28(2), 201-213.

6. Bollaert, E., Erpicum S., Pirotton, M. and Schleiss, A. (2002). "Genetic Algorithm Optimization of Transient TwoPhase Water Pressures Inside Closed-End Rock Joints". In: BOLlaERT, E. (ed.), Rock Scour due to falling High-Velocity Jets-Proc. Int. Workshop, Lausanne, Switzerland, 25-28 September 2002, Balkema. 
7. BÜRgiSSER, M.F. and RutSChMANN, P. (1999). "Numerical Solution of Viscous 2DV Free Surface Flows: Flow Over Spillway Crests". In: 28th IAHR Congress, Graz, Austria.

8. Dewals, B., Archambeau, P., Erpicum, S., Mouzelard, T. and Pirotton, M. (2002). "Coupled Computations of Highly Erosive Flows with WOLF Software". In: Proc. 5th Int. Conf. on Hydro-Science \& Engineering, Warsaw.

9. Dressler, R.F. (1978). "New Nonlinear Shallow Flow Equations with Curvature". J. Hydraul. Res. 16(3), 205-222.

10. Dressler, R.F. and Yevjevich, V. (1984). "HydraulicResistance Terms Modified for the Dressler Curved-Flow Equations”. J. Hydraul. Res. 22(3), 145-156.

11. IlHaN, M.H. (1994). "Computation of Flow Over Vertically Curved Channels". Masters Thesis, Gradual School of Natural and Applied Sciences, Middle East Technical University, Ankara.

12. KhAn, A.A. and StefFler, P.M. (1996a). "Vertically Averaged and Moment Equations Model for Flow Over Curved Beds". J. Hydraul. Eng.-ASCE 122(1), 3-9.

13. Khan, A.A. and StefFler, P.M. (1996). "Modelling Overfalls Using Vertically Averaged and Moment Equations". J. Hydraul. Eng.-ASCE 122(7), 397-402.
14. Mouzelard, T. (2002). "Contribution à la modélisation desécoulements quasi tridimensionnels instationnaires à surface libre". Doctoral Thesis, Université de Liège, $253 \mathrm{p}$.

15. Pirotton, M. (1994). "Modélisation des discontinuités en écoulement instationnaire à surface libre. Du ruissellement hydrologique en fine lame à la propagation d'ondes consécutives aux ruptures de barrages". Doctoral Thesis, Université de Liège.

16. Sinniger, R. (1989). "Ecoulements Stationnaires". Traité de Génie Civil de l'EPFL, Constructions Hydrauliques, 439 p.

17. Sivakumaran, N.S., Hosking R.J. and Tingsanchali, T. (1981). "Steady Shallow Flow Over a Spillway". J. Fluid Mech. 111, 411-420.

18. Sivakumaran, N.S., Tingsanchali, T and Hosking, R.J. (1983). "Steady Shallow Flow Over Curved Beds". J. Fluid Mech. 128, 469-487.

19. US Bureau of Reclamation (1987). Design of Small Dams, 3rd edn. Water Resources Technical Publication, USA.

20. Whitham, G.B. (1974). Linear and Nonlinear Waves. John Wiley \& Sons, New York, 636 p.

21. World Commission on Dams (2000). Dams and Development, A New Framework for Decision-Making. Earthscan, London. 